\title{
A SIMPLE GEOMETRIC CONSTRUCTION INVOLVING ULTRARADICALS
}

\author{
J. ROBERTSON and C. SNYDER ${ }^{\bowtie}$ \\ (Received 25 August 2010; accepted 15 May 2011) \\ Communicated by M. G. Cowling \\ In memory of Curt Meyer (19 November 1919-18 April 2011)
}

\begin{abstract}
We give a new type of geometric construction that allows for the construction of families of quintic irrationalities, and is quite rich in algebraic properties. This construction may be considered as our first attempt at characterizing points constructible with compass and twice-notched ruler, a problem that seems to have been known in some form for more than two millennia.
\end{abstract}

2010 Mathematics subject classification: primary 51M15.

Keywords and phrases: constructible number, marked-ruler construction, quintic extension, ultraradical.

\section{Introduction}

Geometric constructions have been a source of fascination since antiquity. Euclid gave interesting and nontrivial constructions using only the familiar tools of a straightedge and compass. The ancient Greeks must have been sure that certain constructions were impossible, such as trisecting a given angle or duplicating a cube; however, a complete characterization of constructible points in a coordinate plane had to wait until more modern times, after analytic geometry and algebra, in particular field theory, had been invented and well established.

On the other hand, the ancient Greeks were not afraid of extending the assortment of tools they used so as to create more types of constructions. For example, one way uses intersections of conics, which allowed for trisection of angles. Another method uses a marked ruler instead of a straightedge. A characterization of the constructible points is known in some instances but not in others, depending on the tools used. See below for more details.

Here is some motivation for the work presented here. Suppose that we can use a twice-notched ruler (or marked ruler for short), that is, a straightedge with two

(C) 2011 Australian Mathematical Publishing Association Inc. 1446-7887/2011 \$16.00 
marks one unit apart, and a compass. See [1, 4] for a description of this type of construction. If we start with the points $(0,0)$ and $(1,0)$, then it is an open problem to give an algebraic characterization of the points that can be constructed by these tools. For example, it is not known if there is a way to construct the real $\sqrt[5]{2}$ or to quinsect a given angle, even though there are numbers satisfying irreducible quintic polynomials over $\mathbb{Q}$ constructible using only these tools. In this note we give a new type of construction that has a rich set of constructible points, including many whose coordinates are roots of quintic polynomials, but for which an algebraic characterization of the constructible points is much more manageable than for a twicenotched ruler and compass. We do not know if our modified construction process is subsumed under that of the twice-notched ruler and compass, but we would guess that it is. In any case, perhaps surprisingly, all real fifth roots of rational numbers (among many others) are constructible by our modified method.

\section{Tools for q-constructions}

Here, we set up our construction, and define constructible points and numbers.

We shall be working in $\mathbb{R} \times \mathbb{R}$. We call a point in $\mathbb{R} \times \mathbb{R}$-constructible if it is the last point in a finite sequence of points $P_{1}, P_{2}, \ldots, P_{n}$ such that the point is in the 'starter' set

$$
\{(0,0),(1,0),(0,1)\},
$$

or is obtained inductively in one of the following ways:

(i) as the intersection of two lines, each of which passes through two points that appear earlier in the sequence;

(ii) as an intersection of a line passing through two earlier points and a circle passing through $(0,0)$ and centered at a point on the $x$-axis appearing earlier in the sequence;

(iii) as a point of intersection of the graph of $y=x^{3}$ and a line described in (i);

(iv) as a point of intersection of the graph of $y=x^{3}$ and a circle described in (ii).

A real number will be called $q$-constructible, if it is the $x$-coordinate of a qconstructible point lying on the $x$-axis. A line passing through two q-constructible points will be called a $q$-constructible line. Also, for convenience, we call the sequence of points $P_{1}, \ldots, P_{n}$ in the definition a $q$-constructible sequence.

Here are some reasons for looking at this particular type of construction. First of all, if we use the conic $y=x^{2}$ instead of our cubic $y=x^{3}$ along with a straightedge and compass, then the set of points that are constructible is the same as the set of points obtained by using only a marked ruler. See [1, 4, 8] for more details. The numbers constructible by this process are precisely those that lie in a real 2-3-tower over $\mathbb{Q}$. This means that $a$ is constructible by this process if and only if there exists a sequence of field extensions $K_{0}, K_{1}, \ldots, K_{n}$ such that

$$
\mathbb{Q}=K_{0} \subseteq K_{1} \subseteq \cdots \subseteq K_{n} \subseteq \mathbb{R}
$$


where $\left[K_{j}: K_{j-1}\right]=2$ or 3 , for $j=1, \ldots, n$, and $a \in K_{n}$. Notice in particular that intersecting two conics yields at most four points by Bezout's theorem. Hence the numbers constructed this way satisfy polynomials of degree at most four over fields generated by previously constructed numbers. Thus, by the solutions of cubic and quartic equations by Cardano and Ferrari, it is easily seen that the numbers do indeed lie in real 2-3-towers over $\mathbb{Q}$.

Next, if we allow a marked ruler and compass, then it is not too hard to show that the numbers constructible by this process satisfy equations of degree at most six. See [1] for a very nice presentation of this fact and others. Hence by replacing the marked ruler with the cubic $y=x^{3}$, we would expect solutions to equations of degree at most six. See [8] for other suggestions of cubics. However, by our restriction on the use of the compass, we shall see that the degree is at most five; hence the ' $q$ ' in q-construction stands for 'quintic'.

One of our results gives an algebraic characterization of q-constructible numbers.

A real number a is q-constructible if and only if there exists a sequence of field extensions $K_{0}, K_{1}, \ldots, K_{n}$ with $a \in K_{n}$ such that

$$
\mathbb{Q}=K_{0} \subseteq K_{1} \subseteq \cdots \subseteq K_{n} \subseteq \mathbb{R}
$$

where $\left[K_{j}: K_{j-1}\right] \in\left\{\begin{array}{lll}1 & 2,3 & 5\end{array}\right\}$, for all $j=1, \ldots, n$, and if $\left[K_{j}: K_{j-1}\right]=5$ then $K_{j}=$ $K_{j-1}\left(\sqrt[4]{a_{j-1}}\right)$ where $a_{j-1} \in K_{j-1}$ is the unique real root of the polynomial $x^{5}+x-a_{j-1}$.

\section{A characterization of q-constructible numbers}

As promised, we give a characterization of q-constructible numbers. However, we first introduce some notation and terminology for convenience.

We denote by $\mathbb{F}$ the set of all q-constructible numbers.

If $a$ is a real number, then the unique real root of the polynomial $x^{5}+x-a$ is called the ultraradical of $a$ and is denoted by $\sqrt[*]{a}$. If $a \in F$, where $F$ is a subfield of $\mathbb{R}$, then $F(\sqrt[*]{a})$ will be called an ultraradical extension of $F$.

Let $\mathbb{Q}=K_{0} \subseteq K_{1} \subseteq \cdots \subseteq K_{n} \subseteq \mathbb{R}$ be a tower of field extensions, with $\left[K_{j}: K_{j-1}\right]=1$, 2,3 , or 5 (where $j=12, \ldots, n$ ), such that $K_{j}$ is an ultraradical extension of $K_{j-1}$ if the degree is five. We shall call such an extension a (real) q-tower of $\mathbb{Q}$.

Given this notation, here is a statement of one of our results.

THeORem 1. A real number a lies in $\mathbb{F}$ if and only if $a \in K_{n}$ for some real q-tower $K_{0} \subseteq \cdots \subseteq K_{n}$ of $\mathbb{Q}$.

The proof will be carried out in several steps.

Proposition 2. Let $P_{j}=\left(a_{j}, b_{j}\right)$, for $j=1, \ldots, m$, be a q-constructible sequence of points. Then there is a real $q$-tower $K_{0}, \ldots, K_{n}$ of $\mathbb{Q}$ such that $a_{j}, b_{j} \in K_{n}$ for all $j$.

Proof. We use induction on $m$. For $m=1, P_{1}=\left(a_{1}, b_{1}\right) \in\{(0,0),(1,0),(0,1)\}$ and thus $a_{1}, b_{1} \in \mathbb{Q}=K_{0}$. 
Now assume that $m>1$ and that the proposition holds for any q-constructible sequence of less than $m$ points. Let $P_{1}, \ldots, P_{m}$ be a q-constructible sequence of $m$ points. Hence by the induction hypothesis there is a real q-tower $K_{0}, \ldots, K_{n}$ such that $a_{j}, b_{j} \in K_{n}$ for all $j<m$. Now consider $P_{m}=\left(a_{m}, b_{m}\right)$. Then $P_{m}$ is a point of intersection of curves in four possible ways, which we now consider individually.

Suppose that $P_{m}$ is the intersection of two lines, each passing through two earlier points in the sequence. Then the lines have equations with coefficients in $K_{n}$, and thus the coordinates of the point of intersection are in $K_{n}$.

Next suppose that $P_{m}$ is a point of intersection of a line passing through two earlier points in the sequence and a circle passing through $(0,0)$ and centered at a point on the $x$-axis appearing earlier in the sequence. Then the line and circle have equations with coefficients in $K_{n}$, and so $a_{m}, b_{m} \in K_{n}(\sqrt{c})$ for some $c \in K_{n}$ with $c>0$. Hence $a_{m}, b_{m} \in K_{n+1}=K_{n}(\sqrt{c})$, and $\left[K_{n+1}: K_{n}\right]=1$ or 2 , and $K_{n+1} \subseteq \mathbb{R}$. Thus $a_{m}, b_{m} \in K_{n+1}$, the terminal field of a real q-tower of $\mathbb{Q}$.

Now suppose that $P_{m}$ is a point of intersection of the curve $y=x^{3}$ and a line passing through two points appearing earlier in the sequence. As above, the line has an equation with coefficients in $K_{n}$. Thus $a_{m}$ is a real root of a cubic polynomial with coefficients in $K_{n}$. Let $K_{n+1}=K_{n}\left(a_{m}\right)$. Then $K_{n+1} \subseteq \mathbb{R}$ and $\left[K_{n+1}: K_{n}\right] \leq 3$. Moreover $b_{m} \in K_{n+1}$, since $b_{m}=a_{m}^{3}$.

Finally, suppose that $P_{m}$ is a point of intersection of the curve $y=x^{3}$ and the circle passing through $(0,0)$ and with center $P_{j}=\left(a_{j}, 0\right)$ for some $j<m$. Hence $a_{j} \in K_{n}$. The circle has an equation $y^{2}+x^{2}-2 a_{j} x=0$. Thus $a_{m}$ must satisfy the sextic equation $x^{6}+x^{2}-2 a_{j} x=0$. If $a_{m}=0$, then $P_{m}=(0,0)$ and we are done. If not, then $a_{m}$ satisfies the quintic equation

$$
x^{5}+x-a=0,
$$

where $a=2 a_{j} \in K_{n}$. Hence $a_{m}=\sqrt[*]{a}$. So if we let $K_{n+1}=K_{n}(\sqrt[*]{a})$, then $\left[K_{n+1}: K_{n}\right] \leq 5$. We shall be done if we can show $\left[K_{n+1}: K_{n}\right] \neq 4$. Assume otherwise, then

$$
x^{5}+x-a=p(x) q(x)
$$

where $p$ and $q$ are irreducible polynomials over $K_{n}$ of degrees four and one respectively. By assumption, $\sqrt[x]{a}$ is a root of $p$. However, $q$ also has a real root, implying $\sqrt[4]{a}$ must be this root, since the real root of $x^{5}+x-a$ is unique. This is the desired contradiction, and the proposition is now established.

From this proposition, we immediately obtain the following result.

CorollaRy 3. If $a \in \mathbb{F}$, then $a \in K_{n}$ for some real $q$-tower $K_{0}, \ldots, K_{n}$ of $\mathbb{Q}$.

Now we consider the converse. However, we first isolate a useful lemma that follows essentially by observing that one can prove $\mathbb{F}$ is a field by using only a straightedge.

LeMma 4. The set $\mathbb{F}$ is a subfield of $\mathbb{R}$. 
Proof. We just sketch the argument. First note that the points $(1,0),(0,1),(2,0)$ and $(0,2)$ are q-constructible, for the first two are in our starter set; $(2,0)$ and $(1,1)$ are intersections of the (q-constructible) $x$-axis and the curve $y=x^{3}$ respectively with the circle through $(0,0)$ centered at $(1,0)$. However, $(0,2)$ is then the intersection of the qconstructible lines $x+y=2$ and the $y$-axis. This new starter set is all that is necessary to show that the numbers constructible from this set by using only a straightedge form a field; see [4, Ch. 4].

We now take advantage of Martin's presentation. In [4, Ch. 4], starting with Theorem 4.4 through Corollary 4.11, replace the words 'ruler point' and 'ruler line' with 'q-constructible point' and 'q-constructible line' respectively. The proofs of all the results remain unchanged and the new Corollaries 4.9 and 4.11 show that $\mathbb{F}$ is a field. For later use, we note that by the new Theorem 4.7, any line through a q-constructible point and parallel to a q-constructible line is a q-constructible line.

For a detailed proof of this lemma, see [6].

We now use this lemma and its proof to help prove the converse to Corollary 3.

Proposition 5. Let $K_{0}, \ldots, K_{n}$ be a real $q$-tower of $\mathbb{Q}$. Then $K_{n} \subseteq \mathbb{F}$.

Proof. We proceed by induction on $n$. If $n=0$, then $K_{n}=\mathbb{Q} \subseteq \mathbb{F}$, since $\mathbb{F}$ is a field.

Now suppose that $n>1$ and that all q-towers of $\mathbb{Q}$ of length less than $n$ are contained in $\mathbb{F}$. Let $K_{0}, \ldots, K_{n}$ be a real q-tower of $\mathbb{Q}$. Then by the induction hypothesis, $K_{n-1} \subseteq \mathbb{F}$. We consider three cases depending on the degree $\left[K_{n}: K_{n-1}\right]$.

First, suppose that $\left[K_{n}: K_{n-1}\right]=2$. Then $K_{n}=K_{n-1}(\sqrt{a})$ for some $a \in K_{n-1}$ with $a>0$. We claim that $\sqrt{a}$ is q-constructible. The argument is essentially the same as for the Poncelet-Steiner theorem, which shows (roughly) that points constructible by straightedge and one circle are precisely those constructible by the usual straightedge and compass; see [4, Ch. 6]. Thus square roots can be constructed.

Notice that $a>0$, and so $-1<(a-1) /(a+1)<1$ and $0<(a-1) /(a+1)+1<2$. Since $a \in \mathbb{F}$ by the induction hypothesis, $(a-1) /(a+1)+1 \in \mathbb{F}$, as $\mathbb{F}$ is a field. By the last statement in the proof of the lemma, the vertical line through the point $((a-1) /(a+1)+1,0)$ is q-constructible. Hence the points

$$
\left(\frac{a-1}{a+1}+1, \pm \sqrt{1-\left(\frac{a-1}{a+1}\right)^{2}}\right)
$$

of intersection of this line with the circle passing through $(0,0)$ centered at $(1,0)$ (thus with an equation $\left.y^{2}+(x-1)^{2}=1\right)$ are q-constructible. However, by the last statement of the proof of the lemma again, by projecting to the $y$-axis and then along a line of slope -1 we then see that

$$
\sqrt{1-\left(\frac{a-1}{a+1}\right)^{2}} \in \mathbb{F}
$$


However, we now have

$$
\sqrt{a}=\left(\frac{a+1}{2}\right) \sqrt{1-\left(\frac{a-1}{a+1}\right)^{2}} \in \mathbb{F}
$$

as desired.

Next suppose that $\left[K_{n}: K_{n-1}\right]=3$. Then $K_{n}=K_{n-1}(\alpha)$, where $\alpha$ is the real root of an irreducible cubic polynomial $p$ over $K_{n-1}$. We claim that $\mathbb{F}$ is Vietian; that is, if $a \in \mathbb{F}$ with $a>0$, then $\sqrt{a}, \sqrt[3]{a} \in \mathbb{F}$, and, moreover, if $\cos \theta \in \mathbb{F}$, then $\cos (\theta / 3) \in \mathbb{F}$. To see this, suppose that $a \in \mathbb{F}$ with $a>0$; notice from the previous argument that $\sqrt{a} \in \mathbb{F}$. Moreover, $(\sqrt[3]{a}, a)$ is the intersection of the q-constructible line $y=a$ with $y=x^{3}$ and thus q-constructible. Projecting to the $x$-axis shows $\sqrt[3]{a} \in \mathbb{F}$. Finally, let $a=2 \cos \theta \in \mathbb{F}$. Then $x=2 \cos (\theta / 3)$ satisfies the equation $x^{3}-3 x-a=0$. However, this is the $x$ coordinate of the intersection of the line $y=3 x+a$, which is q-constructible, and the curve $y=x^{3}$. Thus $2 \cos (\theta / 3)$ and hence also $\cos (\theta / 3)$ are in $\mathbb{F}$. Therefore, $\mathbb{F}$ is Vietian as claimed. However, by [4, Theorem 9.8], which states that a real root of any polynomial of degree less than five over a Vietian field lies in this field, we then have $\alpha \in \mathbb{F}$. Therefore, since $\mathbb{F}$ is again a field, $K_{n} \subseteq \mathbb{F}$.

Finally, suppose that $\left[K_{n}: K_{n-1}\right]=5$. Then $K_{n}=K_{n-1}(\alpha)$ where $\alpha=\sqrt[*]{a}$ for some $a \in K_{n-1} \subseteq \mathbb{F}$; that is, $\alpha$ is the unique real root of $x^{5}+x-a$. However, the points of intersection of $y=x^{3}$ with the circle passing through $(0,0)$ centered at $(a / 20)$ are then q-constructible. These points are easily seen to be $(0,0)$ and $\left(\alpha, \alpha^{3}\right)$. By projection, $\alpha \in \mathbb{F}$. Therefore $K_{n} \subseteq \mathbb{F}$, as desired.

This establishes the proposition.

Corollary 3 and Proposition 5 establish the theorem, that is, an algebraic characterization of the q-constructible numbers.

\section{More properties and some examples of q-constructible numbers}

We saw in the proof of Proposition 5 that the field $\mathbb{F}$ of q-constructible numbers is Vietian, and so any real root of a polynomial over $\mathbb{F}$ of degree at most four is also q-constructible. Now we address the problem of the q-constructibility of real roots of quintic polynomials over $\mathbb{F}$. First we show that $\mathbb{F}$ is closed under taking fifth roots.

THEOREM 6. If $r \in \mathbb{F}$, then the real fifth root $\sqrt[5]{r} \in \mathbb{F}$.

Hence, as noted in the introduction, all fifth roots of rational numbers are qconstructible.

Note that it suffices to prove the theorem for positive $r$. Moreover, we need only prove the theorem when $r \geq 2$ (or $r$ is sufficiently large), since we can reduce the problem to this case by multiplying by a fifth power of a sufficiently large integer.

We prove the theorem in several steps. In what follows, if $r \in \mathbb{R}$, then $\sqrt[5]{r}$ always means the real fifth root. 
Proposition 7. Suppose that $K$ is a subfield of $\mathbb{R}$ and a finite extension of $\mathbb{Q}$. Suppose, too, that $r \in K, r \geq 2$ and the polynomial $x^{5}-r$ is irreducible in $K[x]$. Then there exists an extension $L$ in $\mathbb{R}$ of $K$ of degree at most three, and there are numbers $a, b \in L$ with $a>0$, such that if $\beta$ is the real root of the polynomial $x^{5}+a x+b$, then $L(\sqrt[5]{r})=L(\beta)$.

Proof. Let $\alpha=\sqrt[5]{r}$. Then $1, \alpha, \alpha^{2}, \alpha^{3}, \alpha^{4}$ is a basis of $L(\alpha) / L$ (where $L$ will be determined below). Let $\beta=a_{0}+a_{1} \alpha+\cdots+a_{4} \alpha^{4}$ for some $a_{j} \in L$. We shall determine the $\operatorname{root} \beta$ described in the statement of the proposition. Since $x^{5}-r=\prod_{j=1}^{5}\left(x-\alpha \zeta^{j}\right)$, where $\zeta=\zeta_{5}$ is a primitive fifth root of unity in $\mathbb{C}$, the minimal polynomial $p$ of $\beta$ over $L$ is given as $p(x)=\prod_{j=1}^{5}\left(x-\beta_{j}\right)$, where $\beta_{j}=\sum_{\mu=0}^{4} a_{\mu} \zeta^{j \mu} \alpha^{\mu}$. Also,

$$
p(x)=x^{5}-\sigma_{1} x^{4}+\sigma_{2} x^{3}-\sigma_{3} x^{2}+\sigma_{4} x-\sigma_{5},
$$

where the $\sigma_{j}$ are the elementary symmetric functions of $\beta_{1}, \ldots, \beta_{5}$, that is,

$$
\sigma_{j}\left(x_{1}, \ldots, x_{5}\right)=\sum_{1 \leq k_{1}<\cdots<k_{j} \leq 5} x_{k_{1}} \cdots x_{k_{j}}
$$

for $j=1, \ldots, 5$, where $x_{k}=\beta_{k}$.

Since $p(x)=x^{5}+a x+b$, we want $\sigma_{1}=\sigma_{2}=\sigma_{3}=0$. Instead of working directly with the $\sigma$, it is easier to use the following power sums of the roots. Let

$$
s_{j}=s_{j}\left(\beta_{1}, \ldots, \beta_{5}\right)=\sum_{k=1}^{5} \beta_{k}^{j} .
$$

Relations between the elementary symmetric functions and the power sums are given by Newton's identities, see [3]:

$$
s_{j}-\sigma_{1} s_{j-1}+-\cdots+(-1)^{j-1} \sigma_{j-1} s_{1}+(-1)^{j} j \sigma_{j}=0,
$$

for $j=1, \ldots, 5$.

Notice that $\sigma_{1}=\sigma_{2}=\sigma_{3}=0$ if and only if $s_{1}=s_{2}=s_{3}=0$. Given the latter, we derive relations among $a_{0}, \ldots, a_{4}$ defined above.

We start with

$$
s_{1}=0
$$

Notice then that

$$
s_{1}=\sum_{i=1}^{5} \beta_{i}=\sum_{i=1}^{5} \sum_{\mu=0}^{4} a_{\mu} \zeta^{i \mu} \alpha^{\mu}=\sum_{\mu=0}^{4} a_{\mu} \alpha^{\mu} \sum_{i=1}^{5} \zeta^{i \mu}=5 a_{0},
$$

since $\sum_{i=1}^{5} \zeta^{i \mu}$ is equal to 0 if $\mu \not \equiv 0 \bmod 5$, but is equal to 5 otherwise. Therefore,

$$
a_{0}=0 \text {, }
$$

which we assume from now on.

Now we consider

$$
s_{2}=0 \text {. }
$$


In this case,

$$
\begin{aligned}
s_{2} & =\sum_{i=1}^{5} \beta_{i}^{2}=\sum_{i=1}^{5}\left(\sum_{\mu=1}^{4} a_{\mu} \zeta^{i \mu} \alpha^{\mu}\right)^{2} \\
& =\sum_{i=1}^{5}\left(\sum_{\mu=1}^{4} a_{\mu}^{2} \alpha^{2 \mu} \zeta^{2 \mu i}+2 \sum_{1 \leq \mu<v \leq 4} a_{\mu} a_{\nu} \alpha^{\mu+v} \zeta^{(\mu+v) i}\right) \\
& =\sum_{\mu=1}^{4} a_{\mu}^{2} \alpha^{2 \mu} \sum_{i=1}^{5} \zeta^{2 \mu i}+2 \sum_{1 \leq \mu<v \leq 4} a_{\mu} a_{v} \alpha^{\mu+v} \sum_{i=1}^{5} \zeta^{(\mu+v) i} \\
& =5\left(2 a_{1} a_{4}+2 a_{2} a_{3}\right) \alpha^{5}=5\left(2 a_{1} a_{4}+2 a_{2} a_{3}\right) r,
\end{aligned}
$$

since $\sum_{i=1}^{5} \zeta^{2 \mu i}=0$ for $\mu=1, \ldots, 4$ and $\sum_{i=1}^{5} \zeta^{(\mu+v) i}=0$, unless $\mu+v$ is a multiple of 5 . From this we obtain

$$
a_{1} a_{4}+a_{2} a_{3}=0
$$

Next we consider

$$
s_{3}=0
$$

Here

$$
\begin{aligned}
s_{3}= & \sum_{i=1}^{5} \beta_{i}^{3}=\sum_{i=1}^{5}\left(\sum_{\mu=1}^{4} a_{\mu} \zeta^{i \mu} \alpha^{\mu}\right)^{3} \\
= & \sum_{\mu=1}^{4} a_{\mu}^{3} \alpha^{3 \mu} \sum_{i=1}^{5} \zeta^{3 \mu i}+3 \sum_{\substack{1 \leq \mu, v \leq 4 \\
\mu \neq \nu}} a_{\mu}^{2} a_{\nu} \alpha^{2 \mu+\nu} \sum_{i=1}^{5} \zeta^{(2 \mu+v) i} \\
& +6 \sum_{1 \leq \mu<v<\varkappa \leq 4} a_{\mu} a_{\nu} a_{\varkappa} \alpha^{\mu+\nu+\varkappa} \sum_{i=1}^{5} \zeta^{(\mu+v+\varkappa) i} \\
= & 15\left(\left(a_{1}^{2} a_{3}+a_{1} a_{2}^{2}\right) \alpha^{5}+\left(a_{3}^{2} a_{4}+a_{4}^{2} a_{2}\right) \alpha^{10}\right),
\end{aligned}
$$

since the first and third sums are zero and the second is nonzero only when $(\mu, v) \in$ $\{(1,3),(3,4),(2,1),(4,2)\}$. Now using the fact that $\alpha^{5}=r$ and the assumption that $s_{3}=0$, we obtain

$$
a_{1} a_{2}^{2}+a_{1}^{2} a_{3}+\left(a_{2} a_{4}^{2}+a_{3}^{2} a_{4}\right) r=0 .
$$

Now we need to ensure that $a>0$. Notice that $a=\sigma_{4}$, and by Newton's identity, with $s_{1}=s_{2}=s_{3}=0$, it follows that $s_{4}+4 \sigma_{4}=0$ and thus

$$
a=-\frac{1}{4} s_{4} .
$$

We now compute $s_{4}$ in terms of the $a_{i}$. Notice that

$$
s_{4}=\sum_{i=1}^{5} \beta_{i}^{4}=\sum_{i=1}^{5}\left(\sum_{\mu=1}^{4} a_{\mu} \zeta^{i \mu} \alpha^{\mu}\right)^{4}=S_{1}+S_{2}+S_{3}+S_{4}+S_{5}
$$


where some calculation shows that

$$
\begin{aligned}
& S_{1}=\sum_{\mu=1}^{4} a_{\mu}^{4} \alpha^{4 \mu} \sum_{i=1}^{5} \zeta^{4 \mu i}=0, \\
& S_{2}=4 \sum_{\mu \neq \nu} a_{\mu}^{3} a_{\nu} \alpha^{3 \mu+\nu} \sum_{i=1}^{5} \zeta^{(3 \mu+v) i}=20\left(a_{1}^{3} a_{2} r+\left(a_{2}^{3} a_{4}+a_{1} a_{3}^{3}\right) r^{2}+a_{3} a_{4}^{3} r^{3}\right), \\
& S_{3}=6 \sum_{\mu<v} a_{\mu}^{2} a_{v}^{2} \alpha^{2(\mu+v)} \sum_{i=1}^{5} \zeta^{2(\mu+v)}=30\left(a_{1}^{2} a_{4}^{2}+a_{2}^{2} a_{3}^{2}\right) r^{2}, \\
& S_{4}=12 \sum_{|\{\mu, \nu, \gamma\}|=3} a_{\mu}^{2} a_{\nu} a_{\varkappa} \alpha^{2 \mu+\nu+\varkappa} \sum_{i=1}^{5} \zeta^{(2 \mu+v+\varkappa) i}=0,
\end{aligned}
$$

and, finally,

$$
S_{5}=24 a_{1} a_{2} a_{3} a_{4} \alpha^{10} \sum_{i=1}^{5} \zeta^{10 i}=120 a_{1} a_{2} a_{3} a_{4} r^{2} .
$$

Therefore, $-4 a=s_{4}$, and this is equal to

$$
10 r\left(2 a_{1}^{3} a_{2}+r\left(2 a_{2}^{3} a_{4}+2 a_{1} a_{3}^{3}+3 a_{1}^{2} a_{4}^{2}+3 a_{2}^{2} a_{3}^{2}+12 a_{1} a_{2} a_{3} a_{4}\right)+2 a_{3} a_{4}^{3} r^{2}\right) .
$$

Summarizing what we have so far,

$$
\beta=a_{1} \alpha+a_{2} \alpha^{2}+a_{3} \alpha^{3}+a_{4} \alpha^{4}
$$

is a root of $p(x)=x^{5}+a x+b$, if the $a_{i}$ satisfy the two conditions,

$$
\begin{gathered}
a_{1} a_{4}+a_{2} a_{3}=0, \\
a_{1} a_{2}^{2}+a_{1}^{2} a_{3}+\left(a_{2} a_{4}^{2}+a_{3}^{2} a_{4}\right) r=0 .
\end{gathered}
$$

Now let $a_{3}=-1$ and $a_{4}=1$; thus by (1), $a_{1}=a_{2}$. Hence by (2),

$$
a_{1}^{3}-a_{1}^{2}+\left(a_{1}+1\right) r=0 \text {. }
$$

However, we then have

$$
s_{4}=10 r\left(2 a_{1}^{4}+\left(2 a_{1}^{3}-6 a_{1}^{2}-2 a_{1}\right) r-2 r^{2}\right) .
$$

Therefore in order that $a>0$, we need $s_{4}<0$ or, equivalently, that

$$
c=r^{2}+\left(a_{1}+3 a_{1}^{2}-a_{1}^{3}\right) r-a_{1}^{4}>0 .
$$

Now notice that if $f(x)=x^{3}-x^{2}+(x+1) r$, then $f$ is an increasing function of $x \in \mathbb{R}$ (since $r \geq 2$ ) and has its real root $a_{1}$ between -1 and 0 , since $f(-1)=-2$ and $f(0)=r$. However, for these constraints on $a_{1}$, we then see that

$$
c>r^{2}-r-1>0 \text {, }
$$

again since $r \geq 2$. 
Now let $L=K\left(a_{1}\right)$, so $L \subset \mathbb{R}$ and $[L: K] \leq 3$. Moreover, $\beta=a_{1} \alpha+a_{1} \alpha^{2}-\alpha^{3}+\alpha^{4}$ and satisfies the equation $x^{5}+a x+b=0$, where $a, b \in L$, with $a>0$ by construction and $b \neq 0$. Also notice that $L(\alpha)=L(\beta)$. This establishes the proposition.

Now we can complete the proof of Theorem 6.

Proof of Theorem 6. Let $r \in \mathbb{F}$ and assume, without loss of generality, that $r \geq 2$. Hence $r \in K_{n}$ for some real q-tower $K_{0} \subseteq \cdots \subseteq K_{n}$ of $\mathbb{Q}$. Consider the field $K_{n}(\sqrt[5]{r})$. If $\left[K_{n}(\sqrt[5]{r}): K_{n}\right]<5$, then $\sqrt[5]{r} \in \mathbb{F}$, since $\mathbb{F}$ is Vietian (see the proof of Proposition 5). Hence we may assume that this field extension is of degree five and thus $x^{5}-r$ is irreducible in $K_{n}[x]$. Now by the previous proposition, there is an extension $L \subseteq \mathbb{R}$ such that $\left[L: K_{n}\right] \leq 3$ and there is an element $\beta$, which is the real root of a polynomial $x^{5}+a x+b \in L[x]$ with $a>0$, such that $L(\sqrt[5]{r})=L(\beta)$. Next, let $N=L(\sqrt[4]{a})$ and let $\gamma=\beta / \sqrt[4]{a}$ where $\sqrt[4]{a}$ denotes the positive fourth root of $a$. Now $\gamma$ is a root of the polynomial $x^{5}+x+b / \sqrt[4]{a^{5}}$. Let $M=L(\sqrt{a})$ and $R=N(\gamma)$. However,

$$
K_{0} \subseteq \cdots \subseteq K_{n} \subseteq L \subseteq M \subseteq N \subseteq R
$$

is then a real q-tower of $\mathbb{Q}$ such that $\sqrt[5]{r} \in R$, as desired.

Notice that $x^{5}-r$ has exactly one real root for $r \in \mathbb{F}$. This is no accident, as the following theorem indicates.

TheOREM 8. Let $p(x)=x^{5}+a_{1} x^{4}+a_{2} x^{3}+a_{3} x^{2}+a_{4} x+a_{5} \in \mathbb{F}[x]$ and be irreducible over $F=\mathbb{Q}\left(a_{1}, \ldots, a_{5}\right)$. If $p$ has a root in $\mathbb{F}$, then $p$ has exactly one real root.

To prove this theorem we first isolate a lemma.

LemMa 9. Let $K / k$ be a finite Galois extension and $F / k$ an arbitrary extension for which both $K$ and $F$ are subfields of some common field. If $L$ is any field such that $F \cap K \subseteq L \subseteq K$, then the degree $[L: F \cap K]=[L F: F]$.

Proof. As is well known, $K F / F$ is a Galois extension and

$$
\operatorname{Gal}(K / K \cap F) \simeq \operatorname{Gal}(K F / F)
$$

Hence $K$ and $F$ are linearly disjoint over $K \cap F$; see, for instance, [5, Section 20]. However, by transitivity of linear disjointness (again see the above reference), $L$ and $F$ are then linearly disjoint over $K \cap F$. Thus $[L: F \cap K]=[L F: F]$, as desired.

Now we proceed with the proof of the theorem.

Proof of Theorem 8. Write $p$ as $\prod_{j=1}^{5}\left(x-\beta_{j}\right)$ where $\beta_{j} \in \mathbb{C}$. Let $K_{0} \subseteq \cdots \subseteq K_{n}$ be a q-tower of minimal length such that there is a root of $p$ contained in $K_{n}$, that is,

$$
n=\min \left\{m: K_{m} \cap\left\{\beta_{1}, \ldots, \beta_{5}\right\} \neq \emptyset\right\} .
$$

Hence $K_{n-1}$ contains no roots of $p$. Let $N=F\left(\beta_{1}, \ldots, \beta_{5}\right)$ be the splitting field of $p$ over $F$. (Recall that $\operatorname{Gal}(N / F)$ is isomorphic to $C_{5}$, the cyclic group of order 
five, $D_{5}$, the dihedral group of order $10, F_{20}$, the Frobenius group of order $20, A_{5}$, the alternating group of degree five, or $S_{5}$, the full symmetric group; see [2, Section 13.2] for a particularly nice presentation of this and related facts.) We consider two cases according as the degree $\left[N \cap F K_{n-1}: F\right]$ is not or is a multiple of 5 .

Case 1. Suppose that $5 \nmid\left[N \cap F K_{n-1}: F\right]$. Let $\beta=\beta_{j} \in K_{n}$. Since $[F(\beta): F]=5$ but $5 \nmid\left[N \cap F K_{n-1}: F\right]$, we see that $\left[\left(N \cap F K_{n-1}\right)(\beta): N \cap F K_{n-1}\right]=5$. Hence by Lemma 9, $\left[F K_{n-1}(\beta): F K_{n-1}\right]=5$; and thus

$$
\left[K_{n}: K_{n-1}\right] \geq\left[K_{n-1}(\beta): K_{n-1}\right] \geq\left[F K_{n-1}(\beta): F K_{n-1}\right]=5 .
$$

Since $\left[K_{n}: K_{n-1}\right] \leq 5$ we have $\left[K_{n}: K_{n-1}\right]=5$. However, this implies that $K_{n} / K_{n-1}$ is an ultraradical extension and so, in particular, the minimal polynomial of any generator of $K_{n} / K_{n-1}$ must have exactly one real root. Since $\beta$ is such a generator and $p$ is its minimal polynomial over $K_{n-1}$ (as well as $F$ ), $p$ must have exactly one real root, as desired.

Case 2. Suppose that $5 \mid\left[N \cap F K_{n-1}: F\right]$. We show that this case cannot occur. Let $G=\operatorname{Gal}(N / F)$ and consider $G$ identified with a subgroup of $S_{5}$ by fixing an ordering of the roots of $p$. This ordering may be altered for convenience in what follows. We now consider the five possible groups to which $G$ can be isomorphic.

If $G \simeq C_{5}$, then $N=F(\beta)$. However, $N \cap F K_{n-1}=F$, since $K_{n-1}$ contains none of the $\beta_{j}$, contrary to the assumption in this case. Thus $G$ cannot be cyclic of order five.

In the other four instances, we claim first that all the $F_{j}=F\left(\beta_{j}\right)$ are distinct. For otherwise suppose, without loss of generality, that $F_{1}=F_{2}$. Then (see [2]) $G$ contains a 5-cycle $\sigma=(12 i j k)$. Again, without loss of generality, assume that $\sigma=(12345)$. However, we then have $F_{2}=\sigma\left(F_{1}\right)=\sigma\left(F_{2}\right)=F_{3}$, and, furthermore, $F_{3}=\sigma\left(F_{2}\right)=$ $\sigma\left(F_{3}\right)=F_{4}$. Similarly $F_{4}=F_{5}$. Thus all the $F_{j}$ are identical, which implies that $G \simeq C_{5}$; but we have ruled this situation out. Thus these fields are distinct as claimed. Now each of the four groups $D_{5}, F_{20}, A_{5}, S_{5}$ has exactly five subgroups of index 5 in the full group. Hence $\operatorname{Gal}\left(N / F_{j}\right)$ must correspond to these maximal subgroups. Now since no $\beta_{j} \in N \cap F K_{n-1}$, we see that $\operatorname{Gal}\left(N / N \cap F K_{n-1}\right) \nsubseteq \operatorname{Gal}\left(N / F_{j}\right)$ for any $j$.

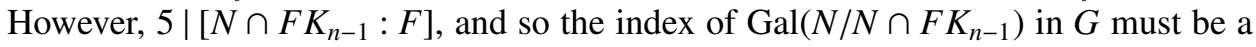
multiple of 5 in $G$, or equivalently the order of $\operatorname{Gal}\left(N / N \cap F K_{n-1}\right)$ is not divisible by 5 .

If $G \simeq D_{5}$ or $F_{20}$, any subgroup whose order is not a multiple of five lies in a subgroup of index 5 in $G$. Thus $\operatorname{Gal}\left(N / N \cap F K_{n-1}\right) \subseteq \operatorname{Gal}\left(N / F_{j}\right)$ for some $j$, contrary to the assumption of Case 2. Hence these two groups cannot occur in this case.

Finally, suppose that $G \simeq S_{5}$ or $A_{5}$. First consider $S_{5}$. The five subgroups of index 5 (hence of order 24) are $S_{4}^{(i)}=\left\{\sigma \in S_{5}: \sigma(i)=i\right\}$, for $i=1, \ldots, 5$. There are subgroups of order relatively prime to five that are not contained in any of the $S_{4}^{(i)}$. They are the conjugates of $H_{1}=\langle(123),(45)\rangle=\langle(123)(45)\rangle \simeq C_{6}$ and $H_{2}=\langle(123),(12)(45)\rangle \simeq S_{3}$, which are of order six, and $H_{3}=\langle(123),(12),(45)\rangle \simeq S_{3} \times S_{2}$ of order 12. It turns out that $H_{3}$ is a maximal subgroup of $S_{5}$ and $H_{3}$ is the only subgroup lying between $S_{5}$ and either of $H_{1}$ and $H_{2}$. Similarly for $A_{5}, H_{2}$ above is a maximal subgroup (unique up to conjugation) in $A_{5}$ not contained in any of the subgroups of index 5 in $A_{5}$. 
Now let $H=\operatorname{Gal}\left(N / N \cap F K_{n-1}\right)$. Then $H$ must be conjugate to $H_{1}, H_{2}$ or $H_{3}$ in $S_{5}$ if $G \simeq S_{5}$, or to $H_{2}$ in $A_{5}$ if $G \simeq A_{5}$. If $G \simeq S_{5}$ and $H$ is conjugate to $H_{3}$ or if $G \simeq A_{5}$ and $H$ is conjugate to $H_{2}$, then $N \cap F K_{n-1} / F$ is of degree 10 with no intermediate subfields. In the other two situations, $\left[N \cap F K_{n-1}: F\right]=20$ and $N \cap F K_{n-1} / F$ contains exactly one intermediate field $K$ and $[K: F]=10$.

Now let $k$ be the maximal index such that $N \cap F K_{k}=F$. Since

$$
F \subset N \cap F K_{k+1} \subseteq N \cap F K_{n-1},
$$

we see that $\left[N \cap F K_{k+1}: F\right] \geq 10$. First suppose that $\left[N \cap F K_{n-1}: F\right]=10$. Thus $N \cap F K_{n-1}=N \cap F K_{k+1}$. However, by Lemma 9, we then have

$$
\left[\left(N \cap F K_{n-1}\right) F K_{k}: F K_{k}\right]=\left[N \cap F K_{n-1}: F\right]=10 .
$$

However,

$$
F K_{k} \subset\left(N \cap F K_{k+1}\right) F K_{k} \subseteq F K_{k+1}
$$

and thus

$$
\left[K_{k+1}: K_{k}\right] \geq\left[F K_{k+1}: F K_{k}\right] \geq 10,
$$

which contradicts the definition of a q-tower. On the other hand, suppose now that $\left[N \cap F K_{n-1}: F\right]=20$. Again, let $K$ be the unique field with $F \subseteq K \subseteq N \cap F K_{n-1}$ for which $[K: F]=10$. Hence $N \cap F K_{k+1}=K$ or $N \cap F K_{k+1}=N \cap F K_{n-1}$ since $F \subset N \cap F K_{k+1} \subseteq N \cap F K_{n-1}$. Applying Lemma 9 again, we see that

$$
\left[\left(N \cap F K_{k+1}\right) F K_{k}: F K_{k}\right] \geq 10 .
$$

However,

$$
F K_{k} \subset K F K_{k} \subseteq\left(N \cap F K_{k+1}\right) F K_{k} \subseteq F K_{k+1},
$$

and so $\left[K_{k+1}: K_{k}\right] \geq 10$, which cannot occur.

This establishes the theorem.

We can say more, namely, the converse of this theorem is also true.

Theorem 10. Let

$$
p(x)=x^{5}+a_{1} x^{4}+a_{2} x^{3}+a_{3} x^{2}+a_{4} x+a_{5} \in \mathbb{F}[x]
$$

and be irreducible over $F=\mathbb{Q}\left(a_{1}, \ldots, a_{5}\right)$. If $p$ has exactly one real root, then this real root is in $\mathbb{F}$.

A major part of the proof of this theorem (in a different context) was given by Sylvester using an application of an extension of his law of inertia for quadratic forms. Here is one version of Sylvester's result, stated in a way that will be convenient.

Theorem 11. Let

$$
p(x)=x^{5}+a_{1} x^{4}+a_{2} x^{3}+a_{3} x^{2}+a_{4} x+a_{5} \in F[x]
$$


with exactly one real root, $\beta$, where $F$ be a subfield of $\mathbb{R}$. Then there are field extensions

$$
F \subseteq K \subseteq L \subseteq M \subseteq \mathbb{R}
$$

such that $[K: F] \leq 2,[L: K] \leq 2$ and $[M: L] \leq 3$ such that $M(\beta)=M(\gamma)$ where $\gamma$ is a root of a polynomial $q(x)=x^{5}-a x-b$ for some $a, b \in M$.

For a proof, see [7]. A similar theorem is true if there is no restriction on the reality of the coefficients. This follows by the (independent) work of Bring and Jerrard as is well known. Sylvester's contribution was in considering the real case.

Before proving Theorem 10, we single out a result; but first we give a couple of definitions. Let

$$
f(x)=a_{0} x^{n}+a_{1} x^{n-1}+\cdots+a_{n}=a_{0} \prod_{i=1}^{n}\left(x-x_{i}\right)
$$

and

$$
g(x)=b_{0} x^{m}+b_{1} x^{m-1}+\cdots+b_{m}=b_{0} \prod_{i=1}^{m}\left(x-y_{i}\right)
$$

be polynomials over some field. Then the discriminant of $f$ is

$$
D(f)=a_{0}^{2 n-2} \prod_{i<j}\left(x_{i}-x_{j}\right)^{2},
$$

and the resultant of $f$ and $g$ is

$$
R(f, g)=a_{0}^{m} b_{0}^{n} \prod_{i=1}^{n} \prod_{j=1}^{m}\left(x_{i}-y_{j}\right) .
$$

Proposition 12. Let $f$ and $g$ be polynomials with real coefficients of degrees three and four respectively, such that $a_{0}>0$ and $b_{0}>0$.

(1) If $D(f)<0$, then exactly one root of $f$ is real.

(2) If $D(g)<0$, then exactly two (distinct) roots of $g$ are real.

(3) Suppose that $D(f)<0$ and $D(g)<0$. Without loss of generality, let $x_{1} \in \mathbb{R}$ and $y_{1}, y_{2} \in \mathbb{R}$ with $y_{1}<y_{2}$. If $R(f, g)<0$, then $y_{1}<x_{1}<y_{2}$, in which case $g\left(x_{1}\right)<0$.

Proof. The statements (1) and (2) are well known (see for example [9, pp. 272-277]). Thus we prove only (3). First,

$$
R(f, g)=a_{0}^{4} b_{0}^{3}\left(x_{1}-y_{1}\right)\left(x_{1}-y_{2}\right) z
$$

where

$$
z=\left(x_{1}-y_{3}\right)\left(x_{1}-y_{4}\right) \prod_{i=2}^{3} \prod_{j=1}^{4}\left(x_{i}-y_{j}\right) .
$$

However, the factors appearing in $z$ come in complex conjugate pairs, and so $z>0$. Further, $\left(x_{1}-y_{1}\right)\left(x_{1}-y_{2}\right)<0$ since $R(f, g)<0$ and $a_{0}, b_{0}>0$. It now follows that $y_{1}<x_{1}<y_{2}$, and hence $g\left(x_{1}\right)<0$. 
Now we prove Theorem 10.

Proof of Theorem 10. It suffices to prove the theorem for $p(x)=x^{5}-a x-b$, by Sylvester's result above. Notice that the case where $a=0$ follows from Theorem 6 . Hence we assume that $a \neq 0$ and consider two cases, according to the sign of $a$.

Case 1. Suppose that $a<0$. Let $\beta$ be the unique real root of $p$. If $c \in \mathbb{R} \backslash\{0\}$, then, since $\beta^{5}-a \beta-b=0$, multiplying this equation by $c^{5}$, we see that

$$
(c \beta)^{5}-a c^{4}(c \beta)-c^{5} b=0 .
$$

Now let $c=1 / \sqrt[4]{|a|}$, where $\sqrt[4]{|a|}$ is one of the real fourth roots of $|a|$. Hence $\beta / \sqrt[4]{|a|}$ satisfies $q(x)=x^{5}+x-b / \sqrt[4]{\left|a^{5}\right|}=0$. Since $|a|^{1 / 4} \in \mathbb{F}$ and thus also $q(x) \in \mathbb{F}[x]$, we see that $\beta /|a|^{5 / 4} \in \mathbb{F}$. However, we then have $\beta \in \mathbb{F}$, as desired.

Case 2. Suppose that $a>0$. The argument in Case 1 then shows that we may assume, without loss of generality, that $p(x)=x^{5}-x-b$. We now follow Bring and Jerrard, and Sylvester, by introducing a (real) Tschirnhaus transformation to transform $p(x)=$ $x^{5}-x-b$ into $q(y)=y^{5}-a_{1} y-b_{1}$, where $a_{1}<0$ and $a_{1}$ and $b_{1}$ are in the appropriate field extension of $F=\mathbb{Q}(b)$. To this end, let $p(x)=x^{5}-x-b=\prod_{i=1}^{5}\left(x-x_{i}\right)$, where $x_{i} \in \mathbb{C}$ are the roots of $p$. Recall that the discriminant $\Delta$ of $p$ is $5^{5} b^{4}-2^{8}$. Since $p$ is irreducible, hence separable, $\Delta \neq 0$. Moreover, since $p$ has exactly one real root, $\Delta>0$; see $[2,9]$. Let

$$
y=u_{0}+u_{1} x+u_{2} x^{2}+u_{3} x^{3}+u_{4} x^{4}
$$

where $u_{i} \in \mathbb{R}$ are to be determined so that

$$
q(y)=y^{5}-a_{1} y-b_{1}=\prod_{i=1}^{5}\left(y-y_{i}\right),
$$

where $y_{i}=\sum_{\mu=0}^{4} u_{\mu} x_{i}^{\mu}$.

Now the coefficients of the polynomials can be expressed in terms of elementary symmetric functions of their roots. However, the coefficients may also be given in terms of power sums of their roots, and for us this will be more convenient, as before. For a nonnegative integer $k$, let

$$
s_{k}=\sum_{i=1}^{5} x_{i}^{k} \quad \text { and } \quad s_{k}^{\prime}=\sum_{i=1}^{5} y_{i}^{k} .
$$

Once again we recall Newton's identities. Let

$$
f(x)=x^{n}+c_{1} x^{n-1}+\cdots+c_{n}=\prod_{i=1}^{n}\left(x-x_{i}\right),
$$

and, for all positive integers $m$, let

$$
s_{m}=\sum_{i=1}^{n} x_{i}^{m}
$$


Then

$$
s_{m}+c_{1} s_{m-1}+\cdots+c_{m-1} s_{1}+m c_{m}=0
$$

where we define $c_{m}=0$ for all $m>n$. From this we can determine the $s_{m}$ recursively.

Again consider $p(x)=x^{5}-x-b$. By Newton's identities (with $c_{1}=c_{2}=c_{3}=0$, $c_{4}=-1$, and $c_{5}=-b$ ), we have for $m=1, s_{1}+c_{1}=0$, and so $s_{1}=-c_{1}=0$. For $m=2$ we see that $s_{2}+c_{1} s_{1}+2 c_{2}=0$ and thus $s_{2}=-c_{1} s_{1}-2 c_{2}=0$. Similarly, $s_{3}=0, s_{4}=4$, and $s_{5}=5 b$. For $m>5$, we see easily that

$$
s_{m}=s_{m-4}+b s_{m-5} .
$$

From all of this we list the first 16 power sums for later use:

$$
\begin{array}{llll}
s_{1}=0, & s_{2}=0, & s_{3}=0, & s_{4}=4, \\
s_{5}=5 b, & s_{6}=0, & s_{7}=0, & s_{8}=4, \\
s_{9}=9 b, & s_{10}=5 b^{2}, & s_{11}=0, & s_{12}=4, \\
s_{13}=13 b, & s_{14}=14 b^{2}, & s_{15}=5 b^{3}, & s_{16}=4 .
\end{array}
$$

Also recall that $q(y)=\prod_{i=1}^{5}\left(y-y_{i}\right)$. We first write

$$
q(y)=y^{5}+B_{1} y^{4}+B_{2} y^{3}+B_{3} y^{2}+B_{4} y+B_{5},
$$

where $y_{i}=\sum_{\mu=0}^{4} u_{\mu} x_{i}^{\mu}$. We want $q(y)=y^{5}-a_{1} y-b_{1}$ as noted above, and so we need to determine the Tschirnhaus transformation such that $B_{1}=B_{2}=B_{3}=0$ and $B_{4}>0$.

By Newton's identities, we may determine the coefficients $B_{j}$ in terms of the power sums $s_{k}^{\prime}$. After this we can rewrite the $s_{k}^{\prime}$ and thus also the $B_{j}$ in terms of $u_{\mu}$ and $s_{m}$ (the power sums of the $x_{i}$ ). To this end, we first want $B_{1}=0$. By Newton's identities,

$$
s_{1}^{\prime}+B_{1}=0
$$

and hence $s_{1}^{\prime}=-B_{1}=0$. However, on the other hand,

$$
\begin{aligned}
s_{1}^{\prime} & =\sum_{i=1}^{5} y_{i}=\sum_{i=1}^{5} \sum_{\mu=0}^{4} u_{\mu} x_{i}^{\mu}=\sum_{\mu=0}^{4} u_{\mu} \sum_{i=1}^{5} x_{i}^{\mu} \\
& =5 u_{0}+u_{1} s_{1}+u_{2} s_{2}+u_{3} s_{3}+u_{4} s_{4}=5 u_{0}+4 u_{4},
\end{aligned}
$$

from our table of values of $s_{m}$ above. Hence

$$
u_{0}=-\frac{4}{5} u_{4}
$$

Thus in order to have $B_{1}=0$, we use the Tschirnhaus transformation:

$$
y=u_{1} x+u_{2} x^{2}+u_{3} x^{3}+u_{4}\left(x^{4}-\frac{4}{5}\right) .
$$

Next, we want $B_{2}=0$. Again using Newton's identities,

$$
s_{2}^{\prime}+B_{1} s_{1}^{\prime}+2 B_{2}=0
$$


and so $s_{2}^{\prime}=-2 B_{2}=0$ (as $\left.B_{1}=0\right)$. On the other hand,

$$
\begin{aligned}
s_{2}^{\prime}= & \sum_{i=1}^{5} y_{i}^{2}=\sum_{i=1}^{5}\left(u_{1} x_{i}+u_{2} x_{i}^{2}+u_{3} x_{i}^{3}+u_{4}\left(x_{i}^{4}-\frac{4}{5}\right)\right)^{2} \\
= & \sum_{i=1}^{5}\left(u_{1}^{2} x_{i}^{2}+u_{2}^{2} x_{i}^{4}+u_{3}^{2} x_{i}^{6}+u_{4}^{2}\left(x_{i}^{8}-\frac{8}{5} x_{i}^{4}+\frac{16}{25}\right)+2\left(u_{1} u_{2} x_{i}^{3}+u_{1} u_{3} x_{i}^{4}\right)\right. \\
& \left.\quad+2\left(u_{1} u_{4}\left(x_{i}^{5}-\frac{4}{5} x_{i}\right)+u_{2} u_{3} x_{i}^{5}+u_{2} u_{4}\left(x_{i}^{6}-\frac{4}{5} x_{i}^{2}\right)+u_{3} u_{4}\left(x_{i}^{7}-\frac{4}{5} x_{i}^{3}\right)\right)\right) \\
= & u_{1}^{2} s_{2}+u_{2}^{2} s_{4}+u_{3}^{2} s_{6}+u_{4}^{2}\left(s_{8}-\frac{8}{5} s_{4}+\frac{16}{5}\right)+2\left(u_{1} u_{2} s_{3}+u_{1} u_{3} s_{4}\right) \\
& \quad+2\left(u_{1} u_{4}\left(s_{5}-\frac{4}{5} s_{1}\right)+u_{2} u_{3} s_{5}+u_{2} u_{4}\left(s_{6}-\frac{4}{5} s_{2}\right)+u_{3} u_{4}\left(s_{7}-\frac{4}{5} s_{3}\right)\right) \\
= & 4 u_{2}^{2}+u_{4}^{2}\left(4-\frac{32}{5}+\frac{16}{5}\right)+2\left(4 u_{1} u_{3}+5 b u_{1} u_{4}+5 b u_{2} u_{3}\right) .
\end{aligned}
$$

Thus we have

$$
0=s_{2}^{\prime}=4 u_{2}^{2}+\frac{4}{5} u_{4}^{2}+8 u_{1} u_{3}+10 b u_{1} u_{4}+10 b u_{2} u_{3} .
$$

Notice that the right side is a quadratic form in the $u_{\mu}$ and can be diagonalized, as was done beautifully by Sylvester in much more generality using his extended version of his law of inertia, by completing the squares as

$$
0=s_{2}^{\prime}=4 v_{2}^{2}+\frac{4}{5} v_{4}^{2}-\frac{25 b^{2}}{4} v_{3}^{2}-\frac{1}{100 b^{2}} \Delta v_{1}^{2},
$$

where

$$
\begin{gathered}
v_{1}=u_{1}, \quad v_{2}=u_{2}+\frac{5 b}{4} u_{3}, \quad v_{3}=u_{3}-\frac{16}{25 b^{2}} u_{1}, \\
v_{4}=u_{4}+\frac{25 b}{4} u_{1}, \quad \text { and } \quad \Delta=5^{5} b^{4}-2^{8},
\end{gathered}
$$

the discriminant of $p$ as identified above. Choosing the $u_{\mu}$ (which we shall do later) to satisfy the above equation will then guarantee that $B_{2}=0$.

Next we want $B_{3}=0$. By Newton's identities,

$$
s_{3}^{\prime}+B_{1} s_{2}^{\prime}+B_{2} s_{1}^{\prime}+3 B_{3}=0
$$

in which case we see that $s_{3}^{\prime}=0$. On the other hand,

$$
s_{3}^{\prime}=\sum_{i=1}^{5} y_{i}^{3}=\sum_{i=1}^{5}\left(\sum_{\mu=0}^{4} u_{\mu} x_{i}^{\mu}\right)^{3} .
$$


Expanding in a manner similar to that in the case of $s_{2}^{\prime}$ above, one finds that

$$
\begin{aligned}
0=s_{3}^{\prime}=- & \frac{12}{25} u_{4}^{3}+9 b u_{3}^{3}+3 b u_{1} u_{4}^{2}+15 b^{2} u_{2} u_{4}^{2}+15 b^{2} u_{3}^{2} u_{4}+12 u_{2} u_{3}^{2} \\
& +\frac{12}{5} u_{2}^{2} u_{4}+15 b u_{1} u_{2}^{2}+15 b u_{1}^{2} u_{3}+12 u_{1}^{2} u_{2}+\frac{24}{5} u_{1} u_{3} u_{4}+30 b u_{2} u_{3} u_{4} .
\end{aligned}
$$

Finally, we need to consider $B_{4}$, which we want to be positive. Again by Newton's identities, we easily see that $s_{4}^{\prime}=-4 B_{4}$, and thus we wish to have $s_{4}^{\prime}<0$. In the same manner as for $s_{2}^{\prime}$ and $s_{3}^{\prime}$, one finds that $s_{4}^{\prime}$ is equal to

$$
\begin{aligned}
& \frac{52}{125} u_{4}^{4}+\left(20 b^{3} u_{3}+8 b^{2} u_{2}+4 b u_{1}\right) u_{4}^{3} \\
& \quad+\left(36 b^{2} u_{3}^{2}+\frac{108}{5} b u_{2} u_{3}+\frac{48}{25} u_{1} u_{3}+\frac{24}{25} u_{2}^{2}+30 b^{2} u_{1}^{2}\right) u_{4}^{2} \\
& \quad+\left(\frac{116}{5} b u_{3}^{3}+\frac{48}{5} u_{2} u_{3}^{2}+120 b^{2} u_{1} u_{2} u_{3}+20 b^{2} u_{2}^{3}+60 b u_{1}^{2} u_{3}+60 b u_{1} u_{2}^{2}+\frac{48}{5} u_{1}^{2} u_{2}\right) u_{4} \\
& \quad+\left(4 u_{3}^{4}+20 b^{2} u_{1} u_{3}^{3}+30 b^{2} u_{2}^{2} u_{3}^{2}+108 b u_{1} u_{2} u_{3}^{2}+36 b u_{2}^{3} u_{3}+24 u_{1}^{2} u_{3}^{2}+48 u_{1} u_{2}^{2} u_{3}\right. \\
& \left.\quad+4 u_{2}^{4}+20 b u_{1}^{3} u_{2}+4 u_{1}^{4}\right) .
\end{aligned}
$$

We are now in a position to choose the $u_{\mu}$ so that $s_{2}^{\prime}=s_{3}^{\prime}=0$ and (perhaps miraculously) $s_{4}^{\prime}<0$. We start by letting

$$
v_{2}=\frac{5}{4} b v_{3} \quad \text { and } \quad v_{4}=\frac{\sqrt{5 \Delta}}{20 b} v_{1},
$$

in which case it is guaranteed that $s_{2}^{\prime}=0$. In terms of the $u_{\mu}$ we see that

$$
u_{2}=\omega_{2} u_{1} \text { and } u_{4}=\omega_{4} u_{1},
$$

with

$$
\omega_{2}=-\frac{4}{5 b} \quad \text { and } \quad \omega_{4}=\frac{\sqrt{5 \Delta}-5^{3} b^{2}}{20 b} .
$$

Notice that $u_{1}$ and $u_{3}$ are still arbitrary.

Now write $u_{3}=\omega_{3} u_{1}$ and compute $s_{3}^{\prime}$ with $u_{\mu}=\omega_{\mu} u_{1}$ for $\mu=2,3,4$. Since $s_{3}^{\prime}$ is a homogeneous form of degree three in $u_{1}, \ldots, u_{4}$, we see that $u_{1}^{3}$ is a factor of $s_{3}^{\prime}$. A straightforward calculation yields

$$
\frac{s_{3}^{\prime}}{3 b u_{1}^{3}}=3 \omega_{3}^{3}+A_{1} \omega_{3}^{2}+A_{2} \omega_{3}+A_{3},
$$

where the $A_{i}=A_{i}(\Delta)$ are given by

$$
\begin{aligned}
A_{1} & =-\frac{16}{5 b^{2}}+\frac{\sqrt{5 \Delta}-5^{3} b^{2}}{4}=-\sqrt{5}\left(\frac{144}{\sqrt{256+\Delta}}+\frac{\Delta}{4 \sqrt{256+\Delta}}-\frac{\sqrt{\Delta}}{4}\right) \\
A_{2} & =45-\frac{8 \sqrt{5 \Delta}}{25 b^{2}}=5\left(9-\frac{8 \sqrt{\Delta}}{\sqrt{256+\Delta}}\right) \\
A_{3} & =-\left(\frac{\sqrt{5 \Delta}-5^{3} b^{2}}{20 b}\right)^{2}\left(2+\frac{\sqrt{5 \Delta}}{5^{3} b^{2}}\right)+\frac{16}{5^{4} b^{4}}\left(\sqrt{5 \Delta}-5^{3} b^{2}\right) \\
& =-5^{3 / 2}\left(\frac{2^{12}(2 \sqrt{\Delta}+3 \sqrt{256+\Delta})}{(256+\Delta)(\sqrt{\Delta}+\sqrt{256+\Delta})^{2}}\right) .
\end{aligned}
$$


Notice that we have given these coefficients in terms of $\Delta$ simply by solving for $b$, which we assume positive without loss of generality. Hence if $\omega_{3}=\omega_{3}(\Delta)$ is a real root of the polynomial

$$
f(x)=f_{\Delta}(x)=3 x^{3}+A_{1} x^{2}+A_{2} x+A_{3},
$$

then $B_{3}=0$.

On the other hand, again letting $u_{\mu}=\omega_{\mu} u_{1}$ for $\mu=2,3,4$ and then setting $x=\omega_{3}$ one finds that

$$
\frac{s_{4}^{\prime}}{u_{1}^{4}}=g(x)=g_{\Delta}(x)=4 x^{4}+C_{1} x^{3}+C_{2} x^{2}+C_{3} x+C_{4},
$$

with $C_{i}=C_{i}(\Delta)$ given as

$$
\begin{aligned}
& C_{1}=\left(\frac{29}{25} \sqrt{\Delta}-\sqrt{256+\Delta}\right) \sqrt{5} \\
& C_{2}=120+\frac{9}{10} \Delta-\frac{1392 \sqrt{\Delta}}{5 \sqrt{256+\Delta}}-\frac{9 \Delta^{3 / 2}}{10 \sqrt{256+\Delta}}, \\
& C_{3}=\frac{87}{\sqrt{5}} \sqrt{\Delta}+\frac{\Delta^{3 / 2}}{4 \sqrt{5}}-\frac{1280 \sqrt{5}}{\sqrt{256+\Delta}}-\frac{119 \Delta}{\sqrt{5} \sqrt{256+\Delta}}-\frac{\Delta^{2}}{4 \sqrt{5} \sqrt{256+\Delta}}, \\
& C_{4}=\frac{25600}{256+\Delta}+\frac{852 \Delta}{256+\Delta}+\frac{25 \Delta^{2}}{8(256+\Delta)}-\frac{464 \sqrt{\Delta}}{\sqrt{256+\Delta}}-\frac{25 \Delta^{3 / 2}}{8 \sqrt{256+\Delta}} .
\end{aligned}
$$

We shall be done essentially if we can show that $g\left(\omega_{3}\right)<0$. To get a hint as to how to proceed we looked numerically at data involving $f$ and $g$ for $\Delta \geq 0$. For $\Delta=0$ (which is, of course, contrary to our assumption), $f(x)=3(x-\sqrt{5})^{3}$ and $g(x)=4(x-\sqrt{5})^{4}$ and thus $f$ and $g$ have the same unique root. On the other hand, for all the positive values of $\Delta$ that we checked, $g$ had exactly two real roots, while $f$ had a unique real root that was sandwiched between the two real roots of $g$. This, of course, implies that $g\left(\omega_{3}\right)<0$ in these cases. To actually prove these facts, in light of Proposition 12 it suffices to show that for all $\Delta>0$, the discriminants of $f$ and $g$ are negative and that the resultant of $f$ and $g$ is also negative. To evaluate these quantities symbolically, we used MATHEMATICA.

The discriminant of $f_{\Delta}$ is found to be

$$
D(f)=\frac{125}{32(256+\Delta)^{5 / 2}}(A-B),
$$

where

$$
A=387 \Delta^{1 / 2}+2 \Delta^{3 / 2} \text { and } B=(131+2 \Delta) \sqrt{256+\Delta} .
$$

However, $D(f)<0$ if and only if $B^{2}-A^{2}>0$. Moreover,

$$
B^{2}-A^{2}=1536 \Delta+4393216>0,
$$

as desired. 
Next we found the discriminant of $g$ to be

$$
D(g)=\frac{27 \Delta^{6}}{31250(256+\Delta)^{3}}(C-D),
$$

where

$$
\begin{aligned}
& C=\left(50697+1036 \Delta+4 \Delta^{2}\right) \sqrt{\Delta} \sqrt{256+\Delta}, \\
& D=2196608+150537 \Delta+1548 \Delta^{2}+4 \Delta^{3} .
\end{aligned}
$$

Hence $D(g)<0$ if and only if $D^{2}-C^{2}>0$. However,

$$
D^{2}-C^{2}=589824 \Delta^{2}+3373989888 \Delta+4825086705664>0,
$$

as desired.

We now consider the resultant of $f$ and $g$. This turns out to be

$$
R(f, g)=\frac{\Delta^{6}}{k \sqrt{256+\Delta}}(E-F),
$$

where

$$
\begin{aligned}
& E=\left(m_{0}+m_{1} \Delta+\cdots+m_{7} \Delta^{7}\right) \sqrt{\Delta} \\
& F=\left(n_{0}+n_{1} \Delta+\cdots+n_{7} \Delta^{7}\right) \sqrt{256+\Delta}
\end{aligned}
$$

where $k$, the $m_{j}$ and the $n_{j}$ are given in Table 1 . Hence $R(f, g)<0$ if and only if $F^{2}-E^{2}>0$. However, it turns out that

$$
F^{2}-E^{2}=k_{0}+k_{1} \Delta+\cdots+k_{11} \Delta^{11},
$$

where the $k_{j}$ are also given in Table 1 . Notice that these coefficients are all positive, as desired.

Finally we can show that the unique real root, $x_{1}$ say, of $p(x)=x^{5}-x-b$ is q-constructible. This will be done by showing that $x_{1}$ lies in a q-tower of $\mathbb{Q}$. To this end, notice that since $b \in \mathbb{F}$, there exists a q-tower of $\mathbb{Q}$, say $K_{0} \subseteq \cdots K_{n}$, for which $b \in K_{n}$. If $p$ is reducible over $K_{n}$, then $x_{1} \in \mathbb{F}$, being the real root of a polynomial over $K_{n}$ of degree at most four, for recall that $\mathbb{F}$ is Vietian. Hence we assume that $p$ is irreducible over $K_{n}$. Now let

$$
u_{1}=1, \quad u_{2}=-\frac{4}{5 b}, \quad u_{4}=\frac{\sqrt{5 \Delta}-5^{3} b^{2}}{20 b},
$$

and $u_{3}=\omega_{3}=\omega_{3}(\Delta)$ be the real root of $f_{\Delta}$. Notice that

$$
f_{\Delta} \in \mathbb{Q}(b, \sqrt{5 \Delta})[x] \text { and } \mathbb{Q}\left(u_{1}, \ldots, u_{4}\right)=\mathbb{Q}\left(b, \sqrt{5 \Delta}, \omega_{3}\right) .
$$


TABLE 1. Relevant coefficients.

\begin{tabular}{|c|c|}
\hline$k$ & 32768000000 \\
\hline$m_{0}$ & 14269936894300127232000000 \\
\hline$m_{1}$ & 570317780839038976000000 \\
\hline$m_{2}$ & 8998789708775424000000 \\
\hline$m_{3}$ & 74991638937600000000 \\
\hline$m_{4}$ & 362223042560000000 \\
\hline$m_{5}$ & 1023543552000000 \\
\hline$m_{6}$ & 1575936000000 \\
\hline$m_{7}$ & 1024000000 \\
\hline$n_{0}$ & 2415196037665783808000000 \\
\hline$n_{1}$ & 203254619999043584000000 \\
\hline$n_{2}$ & 4509367768449024000000 \\
\hline$n_{3}$ & 46721862205440000000 \\
\hline$n_{4}$ & 264570961920000000 \\
\hline$n_{5}$ & 846989568000000 \\
\hline$n_{6}$ & 1444864000000 \\
\hline$n_{7}$ & 1024000000 \\
\hline$k_{0}$ & 1493292006491264562842625502974715101184000000000000 \\
\hline$k_{1}$ & 53542746396833318735872129176912789504000000000000 \\
\hline$k_{2}$ & 857182471590562824743459659626577920000000000000 \\
\hline$k_{3}$ & 8056613010345157895879306206248960000000000000 \\
\hline$k_{4}$ & 49136561411116580536626519736320000000000000 \\
\hline$k_{5}$ & 202673055899025936195212279808000000000000 \\
\hline$k_{6}$ & 570656643744086383518549968000000000000 \\
\hline$k_{7}$ & 1078586086986262008299520000000000000 \\
\hline$k_{8}$ & 1304721582145362984960000000000000 \\
\hline$k_{9}$ & 916870188991774720000000000000 \\
\hline$k_{10}$ & 310178243149824000000000000 \\
\hline$k_{11}$ & 38654705664000000000000 \\
\hline
\end{tabular}

Now let

$$
y_{1}=x_{1}-\frac{4}{5 b} x_{1}^{2}+\omega_{3}(\Delta) x_{1}^{3}+\frac{\sqrt{5 \Delta}-5^{3} b^{2}}{20 b}\left(x_{1}^{4}-\frac{4}{5}\right) \in \mathbb{Q}\left(b, \sqrt{5 \Delta}, \omega_{3}, x_{1}\right) .
$$

By our construction, $y_{1}$ is the real root of $q(y)=y^{5}+B_{4} y+B_{5}$, where $B_{4}>0$ and $B_{4}, B_{5} \in \mathbb{Q}\left(b, \sqrt{5 \Delta}, \omega_{3}\right)$. However, $y_{1} / B_{4}^{1 / 4}$ is then the real root of $y^{5}+y+B_{5} / B_{4}^{5 / 4}$, that is,

$$
y_{1}=-B_{4}^{1 / 4} \sqrt[3]{B_{5} B_{4}^{-5 / 4}} .
$$


Hence we have a q-tower

$$
K_{0} \subseteq \cdots \subseteq K_{n} \subseteq K_{n}(\sqrt{5 \Delta}) \subseteq K_{n}\left(\sqrt{5 \Delta}, \omega_{3}\right) \subseteq K_{n}\left(\sqrt{5 \Delta}, \omega_{3}, \sqrt{B_{4}}\right) \subseteq L \subseteq M,
$$

where $L=K_{n}\left(\sqrt{5 \Delta}, \omega_{3}, \sqrt[4]{B_{4}}\right)$ and $M=L\left(\sqrt[*]{B_{5} B_{4}^{-5 / 4}}\right)$ with $y_{1} \in M$. Now observe that $L\left(y_{1}\right) \subseteq L\left(x_{1}\right)$; but $\left[K_{n}\left(x_{1}\right): K_{n}\right]=5$ and since $\left[L: K_{n}\right] \mid 24$ as seen by the q-tower, hence is relatively prime to $5,\left[L\left(x_{1}\right): L\right]=5$. However, from Equation (3) above, $y_{1} \notin L$. Thus $L\left(x_{1}\right)=L\left(y_{1}\right)$ and so $x_{1} \in M \subseteq \mathbb{F}$, as desired.

\section{Some comments and questions}

In case it is not obvious to the reader, we note that q-constructibility cannot possibly coincide with that of a compass and a twice-notched straightedge. For example, the three real roots of the irreducible (2-Eisenstein) polynomial

$$
x^{5}-4 x^{4}+2 x^{3}+4 x^{2}+2 x-6 \in \mathbb{Q}[x]
$$

are constructible with compass and marked ruler (see [1]), but are not q-constructible by Theorem 8 above.

Secondly, even though extracting fifth roots is a q-constructible process, ' $q$ quinsecting' angles is generally not. In particular, q-quinsecting a $90^{\circ}$ angle requires q-constructing an $18^{\circ}$ angle. However, if $\theta=2 \cos 18^{\circ}$, then $\theta$ is a root of the polynomial $x^{5}-5 x^{3}+5 x-4$, which is easily seen to be irreducible over $\mathbb{Q}$ with five real roots.

Next, notice that we restricted the compass to pass through the origin and have center on the $x$-axis, in order to deal with fairly simple polynomials of degree at most five. Had we used an unrestricted compass instead, then we still could not have constructed all possible numbers arising from compass and marked ruler. For the intersection of a circle and $y=x^{3}$ can have at most four points, but on the other hand it is possible to construct the roots of irreducible polynomials of degree at least five with more than four real roots.

On the other hand, if we replace $y=x^{3}$ by other cubic curves, either of genus zero again or elliptic curves, is it possible to characterize the numbers constructible using any one of these curves, along with a straightedge and (unrestricted) compass? Is it possible too that for some cubic curve, this construction process produces the same set of points as that of compass and marked ruler?

\section{Acknowledgements}

The second-named author would like to thank his son-in-law, Todd Berry, for using his free time to set up various software programs, including MATHEMATICA, on the author's laptop. MATHEMATICA was especially useful for the latter part of this paper. Thank you, Todd!

This manuscript is an extension of the first author's Master of Arts thesis at the University of Maine. 


\section{References}

[1] A. Baragar, 'Constructions using a compass and twice-notched straightedge', Amer. Math. Monthly 109 (2002), 151-164.

[2] D. Cox, Galois Theory (Wiley Interscience, Hoboken, NJ, 2004).

[3] D. Dummit and R. Foote, Abstract Algebra, 2nd edn (Prentice Hall, Englewood Cliffs, NJ, 1999).

[4] G. E. Martin, Geometric Constructions (Springer, New York, 1998).

[5] P. Morandi, Field and Galois Theory, Graduate Texts in Mathematics, 167 (Springer, New York, 1996).

[6] J. Robertson, 'A variation on geometric constructions', MA Thesis, University of Maine, Orono, December 2010.

[7] J. J. Sylvester, 'On the so-called Tschirnhausen transformation', J. reine angew. Math. 1887(100) (1887), 465-486.

[8] C. R. Videla, 'On points constructible from conics', Math. Intelligencer 19 (1997), 53-57.

[9] H. Weber, Lehrbuch der Algebra, 2nd edn, Vol. 1 (F. Vieweg, Braunschweig, 1898).

\section{J. ROBERTSON, 1807 County Road, New Limerick, Maine 04761, USA}

e-mail: james.robertson@umit.maine.edu

C. SNYDER, Department of Mathematics and Statistics, University of Maine, Orono, Maine 04469, USA

e-mail: snyder@math.umaine.edu 